\title{
Backtracking games and inflationary fixed points
}

\author{
Anuj Dawar ${ }^{1}$, Erich Grädel ${ }^{2}$, and Stephan Kreutzer ${ }^{3}$ \\ 1 University of Cambridge Computer Laboratory, Cambridge CB3 0FD, UK, \\ anuj.dawar@cl.cam.ac.uk \\ 2 Mathematische Grundlagen der Informatik, Aachen-University, \\ graedel@informatik.rwth-aachen.de \\ 3 Logik in der Informatik, Humboldt-University, Berlin, \\ kreutzer@informatik.hu-berlin.de
}

\begin{abstract}
We define a new class of games, called backtracking games. Backtracking games are essentially parity games with an additional rule allowing players, under certain conditions, to return to an earlier position in the play and revise a choice.

This new feature makes backtracking games more powerful than parity games. As a consequence, winning strategies become more complex objects and computationally harder. The corresponding increase in expressiveness allows us to use backtracking games as model checking games for inflationary fixed-point logics such as IFP or MIC. We identify a natural subclass of backtracking games, the simple games, and show that these are the "right" model checking games for IFP by a) giving a translation of formulae $\varphi$ and structures $\mathfrak{A}$ into simple games such that $\mathfrak{A} \models \varphi$ if, and only if, Player 0 wins the corresponding game and b) showing that the winner of simple backtracking games can again be defined in IFP.
\end{abstract}

\section{Introduction}

The view of logic as a dialectic game, a set of rules by which a proponent attempts to convince an opponent of the truth of a proposition, has deep roots going back to Aristotle. One of the modern manifestations of this view is the presentation of the semantics of logical operators as moves in a two-player game. A paradigmatic example is the Hintikka semantics of first-order logic, which is just one instance of what are now commonly called model-checking games. These are two-player games played on an arena which is formed as the product of a structure $\mathfrak{A}$ and a formula $\varphi$ where one player attempts to prove that $\varphi$ is satisfied in $\mathfrak{A}$ while the other player attempts to refute this.

Model-checking games have proved an especially fruitful area of study in connection with logics for the specification of concurrent systems. The modal $\mu$-calculus $L_{\mu}$ is widely used to express properties of such systems and, in terms of expressive power it subsumes a variety of common modal and temporal logics. The most effective algorithms for model checking properties specified in $L_{\mu}$ are based on parity games. Formally, a parity game is played on an arena $\mathcal{G}:=$ $\left(V, E, V_{0}, V_{1}, \Omega\right)$, where $(V, E)$ is a directed graph, $V_{0}, V_{1} \subseteq V$ form a partition of $V$, and $\Omega: V \rightarrow\{0, \ldots, k-1\}$ assigns to each node a priority. The two 
players move a token around the graph, with Player 0 moving when the token is on a node in $V_{0}$ and Player 1 when it is on $V_{1}$. The edges $E$ determine the possible moves. To determine the winner, we look at the sequence of priorities $\Omega\left(v_{i}\right)$ occurring in an infinite play $v_{0} v_{1} \ldots$ Player 0 wins if the smallest priority occurring infinitely often is even and Player 1 wins if it is odd.

Parity games are the model-checking games not just for $L_{\mu}$ but also of LFPthe extension of first-order logic with an operator for forming relational least fixed points. That is, for any formula $\varphi$ of LFP and any structure $\mathfrak{A}$ one can easily construct a game $\mathcal{G}(\mathfrak{A}, \varphi)$ where Player 0 has a winning strategy if, and only if, the formula $\varphi$ is satisfied in $\mathfrak{A}$. The game arena is essentially obtained as the product of $\mathfrak{A}^{w}$ and $\varphi$, where $w$ is the width of the formula - the maximal arity of a relation defined by a subformula of $\varphi$. Furthermore, for any fixed number $k$, the class of parity games with $k$ priorities in which Player 0 has a winning strategy is itself definable in $L_{\mu}$ and therefore by an LFP formula of width 2 . This tight correspondence between games and the fixed-point logic leads us to describe parity games as the "right" model-checking games for LFP.

LFP is not the only logic that extends first-order logic with a means of forming fixed points. In the context of finite model theory, a rich variety of fixed-point operators has been studied due to the close connection that the resulting logics have with complexity classes. Here we are mainly concerned with IFP, the logic of inflationary fixed points (see Section 3 for a definition). In the context of finite model theory the logics IFP and LFP have often been used interchangeably as it has long been known that they have equivalent expressive power on finite structures. More recently, it has been shown that the two logics are equally expressive even without the restriction to finite structures [6]. However, it has also recently been shown that the extension of propositional modal logic is vastly more expressive than $L_{\mu}$ [1] and that LFP and IFP have very different structural properties even when they have the same expressive power [6]. This exploration of the different nature of the fixed-point operators leads naturally to the question of what an appropriate model-checking game for IFP might look like.

The correspondence between parity games and logics with least and greatest fixed point operators rests on the structural property of well-foundedness. A proponent in a game who is trying to prove that a certain element $x$ belongs to a least fixed point $X$, needs to present a well-founded justification for its inclusion. That is, the inclusion of $x$ in $X$ may be based on the inclusion of other elements in $X$ whose inclusion in turn needs to be justified but the entire process must be well-founded. On the other hand, justification for including an element in a greatest fixed point may well be circular. This interaction between sequences that are required to be finite and those that are required to be infinite provides the structural correspondence with parity games.

A key difference that arises when we consider inflationary fixed points (and, dually, deflationary fixed points) is that the stage at which an element $x$ enters the construction of the fixed point $X$ may be an important part of the justification for its inclusion. In the case of least and greatest fixed points, the operators involved are monotone. Thus, if the inclusion of $x$ can be justified at some stage, 
it can be justified at all later stages. In contrast, in constructing an inflationary fixed point, if $x$ is included in the set, it is on the basis of the immediately preceding stage of the iteration. It may be possible to reflect this fact in the game setting by including the iteration stage as an explicit component of the game position. However, our aim is to leave the notion of the game arena unchanged as the product of the structure and the formula. We wish only to change the rules of the game to capture the nature of the inflationary fixed point operator.

The change we introduce to parity games is that either player is allowed to backtrack to an earlier position in the game, effectively to force a countback of the number of stages. That is, when a backtracking move is played, the number of positions of a given priority that are backtracked are counted and this count plays an important role in the succeeding play. The precise definition is given in Section 3 below. The backtracking games we define are far more complex than parity games. We prove that winning strategies are necessarily more complicated, requiring unbounded memory, in contrast to the memoryless strategies that work for parity games. Furthermore, deciding the winner is PSPACE-hard and remains hard for both NP and Co-NP with only two priorites. In contrast, parity games are known to be decidable in NP $\cap \mathrm{Co}-\mathrm{NP}$ and in PTIME when the number of priorities is fixed. In Section 3 we show that the model-checking problem for IFP can be represented in the form of backtracking games. The construction allows us to observe that a simpler form of backtracking game suffices which we call simple backtracking games. In Section 4 we show that in IFP we can define the class of simple backtracking games that are won by Player 0 . Thus, we obtain a tight correspondence between the game and the logic, as exists between LFP and parity games.

\section{Games with Backtracking}

Backtracking games are essentially parity games with the addition that, under certain conditions, players can jump back to an earlier position in the play. This kind of move is called backtracking.

A backtracking move from position $v$ to an earlier position $u$ is only possible if $v$ belongs to a given set $B$ of backtrack positions, if $u$ and $v$ have the same priority and if no position of smaller priority has occurred between $u$ and $v$. With such a move, the player who backtracks not only resets the play back to $u$, he also commits herself to a backtracking distance $d$, which is the number of positions of priority $\Omega(v)$ that have been seen between $u$ and $v$. After this move, the play ends when $d$ further positions of priority $\Omega(v)$ have been seen, unless this priority is "released" by a lower priority.

For finite plays we have the winning condition that a player wins if her opponent cannot move. For infinite plays, the winner is determined according to the parity condition, i.e., Player 0 wins a play $\pi$ if the least priority seen infinitely often in $\pi$ is even, otherwise Player 1 wins.

Definition 2.1. The arena $\mathcal{G}:=\left(V, E, V_{0}, V_{1}, B, \Omega\right)$ of a backtracking game is a directed graph $(V, E)$, with a partition $V=V_{0} \cup V_{1}$ of $V$ into positions of 
Player 0 and positions of Player 1, a subset $B \subseteq V$ of backtrack positions and a map $\Omega: V \rightarrow\{0, \ldots, k-1\}$ that assigns to each node a priority.

In case $(v, w) \in E$ we call $w$ a successor of $v$ and we denote the set of all successors of $v$ by $v E$. A play of $\mathcal{G}$ from initial position $v_{0}$ is formed as follows. If, after $n$ steps the play has gone through positions $v_{0} v_{1} \ldots v_{n}$ and reached a position $v_{n} \in V_{\sigma}$, then Player $\sigma$ can select a successor $v_{n+1} \in v_{n} E$; this is called an ordinary move. But if $v_{n} \in B$ is a backtrack position, of priority $\Omega\left(v_{n}\right)=q$, say, then Player $\sigma$ may also choose to backtrack; in that case she selects a number $i<n$ subject to the conditions that $\Omega\left(v_{i}\right)=q$ and $\Omega\left(v_{j}\right) \geq q$ for all $j$ with $i<j<n$. The play then proceeds to position $v_{n+1}=v_{i}$ and we set $d(q)=\left|\left\{k: i \leq k<n \wedge \Omega\left(v_{k}\right)=q\right\}\right|$. This number $d(q)$ is relevant for the rest of the game, because the play ends when $d(q)$ further positions of priority $q$ have been seen without any occurrence of a priority $<q$. Therefore, a play is not completely described by the sequence $v_{0} v_{1} \ldots$ of the positions that have been visited. For instance, if a player backtracks from $v_{n}$ in $v_{0} \ldots v_{i} \ldots v_{j} \ldots v_{n}$, it matters whether she backtracks to $i$ or $j$, even if $v_{i}=v_{j}$ because the associated numbers $d(p)$ are different.

We now proceed to a more formal description of how backtracking games are played. We distinguish therefore between the notion of a (partial) play, which is a word $\pi \in(V \cup \mathbb{N}) \leq \omega$ and the sequence path $(\pi)$ of nodes visited by $\pi$. Further, we associate with every partial play $\pi$ a function $d_{\pi}:\{0, \ldots, k-1\} \rightarrow \mathbb{N} \cup\{\infty\}$ associating with every priority $p$ the distance $d_{\pi}(p)$. Here $d(p)=\infty$ means that $p$ is not active; either there never has been a backtracking move of priority $p$, or the priority $p$ has since been released by a smaller priority. Every occurrence of a node with priority $p$ decrements $d_{\pi}(p)$, with the convention that $\infty-1=\infty$. A play $\pi$ cannot be extended if $d_{\pi}(p)=0$ for some $p$.

Definition 2.2 (Playing backtracking games). Let $\mathcal{G}=\left(V, E, V_{0}, V_{1}, B, \Omega\right)$ be a backtracking game with priorities $\{0, \ldots, k-1\}$, and $v_{0} \in V$. The set of partial plays $\pi$ from position $v_{0}$, together with the associated sequence $\operatorname{path}(\pi)$ of the visited positions and the distance function $d_{\pi}:\{0, \ldots, k-1\} \rightarrow \mathbb{N} \cup\{\infty\}$, are inductively defined as follows.

start: $v_{0}$ is a partial play, with path $\left(v_{0}\right)=v_{0}$, and $d_{v_{0}}(p)=\infty$ for all $p$. ordinary move: If $\pi$ is a partial play with $d_{\pi}(p)>0$ for all $p$, $\operatorname{path}(\pi)=$ $v_{0} \ldots v_{n}$ and $v_{n} \in V_{\sigma}$, then Player $\sigma$ can extend $\pi$ to $\pi v$ for each $v \in v_{n} E$; Further, $\operatorname{path}(\pi v)=\operatorname{path}(\pi) v$ and $d_{\pi v}(p):=d_{\pi}(p)$ for $p<\Omega(v), d_{\pi v}(p):=$ $d_{\pi}(p)-1$ for $p=\Omega(v)$, and $d_{\pi v}(p):=\infty$ for $p>\Omega(v)$.

backtracking move: Suppose that $\pi$ is a partial play with $d_{\pi}(p)>0$ for all $p$ and that $\operatorname{path}(\pi)=v_{0} \ldots v_{n}$ with $v_{n} \in V_{\sigma} \cap B, \Omega\left(v_{n}\right)=q$, and $d_{\pi}(q)=\infty$. Then Player $\sigma$ can extend $\pi$ to $\pi i$ for any number $i<n$ such that $\Omega\left(v_{i}\right)=q$ and $\Omega\left(v_{k}\right) \geq q$ for all $k$ with $i<k<n$. Further $\operatorname{path}(\pi i)=\operatorname{path}(\pi) v_{i}$ and $d_{\pi i}(p):=d_{\pi}(p)$ for $p<q, d_{\pi i}(p):=\left|\left\{k: i \leq k<n: \Omega\left(v_{k}\right)=q\right\}\right|$ for $p=q$, and $d_{\pi i}(p):=\infty$ for $p>q$.

Definition 2.3 (Winning condition). A partial play $\pi$ with $\operatorname{path}(\pi)=v_{0} \ldots v_{n}$ is won by Player $\sigma$, if $v_{n} \in V_{1-\sigma}$ and no move is possible. This is the case if 
either $d_{\pi}(p)=0$ for some $p$, or if $v_{n} E$ is empty and no backtracking move is possible from $\pi$. An infinite play $\pi$ is won by Player 0 if the smallest priority occurring infinitely often on $\operatorname{path}(\pi)$ is even; otherwise $\pi$ is won by Player 1 .

A game is determined if from each position one of the two players has a winning strategy. Determinacy of backtracking games follows from general facts on infinite games. Indeed, by Martin's Theorem [7] all Borel games are determined, and it is easy to see that backtracking games are Borel games.

Proposition 2.4. Backtracking games are determined.

Backtracking games generalise parity games. Indeed a parity game is a backtracking game without backtrack positions. Since parity games are determined via positional (i.e. memoryless) winning strategies, the question arises whether this also holds for backtracking games. We present a simple example to show that this is not the case. In fact, no fixed amount of finite memory suffices. For background on positional and finite-memory strategies we refer to [5].

Theorem 2.5. Backtracking games in general do not admit finite-memory winning strategies.

Proof. Consider the following game (where circles are positions of Player 0 and boxes are positions of Player 1).

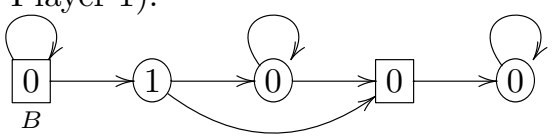

We claim that Player 0 wins from the leftmost position, but needs infinite memory to do so. Clearly, if Player 1 never leaves the leftmost position, or if she leaves it before doing a backtracking move, then Player 0 wins seeing priority 0 infinitely often. If Player 1 at some point backtracks at the leftmost position and then moves on, the strategy of Player 0 depends on the value of $d(0)$ to make sure that the fourth node is hit at the point when $d(0)=0$. But Player 1 can make $d(0)$ arbitrarily large, no finite-memory strategy suffices for Player 0.

This result establishes that winning strategies for backtracking games are more complex than the strategies needed for parity games. It is also the case that the computational complexity of deciding which player has a winning strategy is also higher for backtracking games than for parity games. While it is known that winning regions of parity games can be decided in $\mathrm{NP} \cap \mathrm{Co}-\mathrm{NP}$ (and it is conjectured by many, that this problem is actually solvable in polynomial time), the corresponding problem for backtracking games is PSPACE-hard. Further, for any fixed number of priorities, parity games can be decided in PTIME, but there are examples of backtracking games with just two priorities that are NP-hard. The proof is by reduction from the language equivalence problem for finite automata over a unary alphabet, which is known to be Co-NP-hard [2]. As the problem of deciding the winner of a backtracking game is closed under complementation, it is also NP-hard.

Theorem 2.6. Deciding the winner of backtracking games is Co-NP and NPhard, even for games with only two priorities. 


\section{Model checking games for inflationary fixed point logic}

In this section we want to show that backtracking games can be used as model checking games for inflationary fixed point logics. We will present the games in terms of IFP, the extension of first-order logic by inflationary and deflationary fixed points, but the construction applies, with the obvious modifications, also to the modal iteration calculus MIC [1].

Inflationary fixed point logic. A formula $\varphi(R, \boldsymbol{x})$ with a free $k$-ary secondorder variable and a free $k$-tuple of first-order variables $\boldsymbol{x}$ defines, on every structure $\mathfrak{A}$, a relational operator $F_{\varphi}: \mathcal{P}\left(A^{k}\right) \rightarrow \mathcal{P}\left(A^{k}\right)$ taking $R \subseteq A^{k}$ to the set $\{\boldsymbol{a}:(\mathfrak{A}, R) \models \varphi(\boldsymbol{a})\}$. Fixed point extensions of first-order logic are obtained by adding to FO explicit constructs to form fixed points of definable operators. The type of fixed points that are used determines the expressive power and also the algorithmic complexity of the resulting logics. The most important of these extensions are least fixed point logic (LFP) and inflationary fixed point logic (IFP).

The inflationary fixed point of any operator $F: \mathcal{P}\left(A^{k}\right) \rightarrow \mathcal{P}\left(A^{k}\right)$ is defined

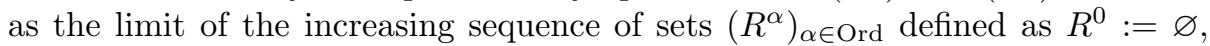
$R^{\alpha+1}:=R^{\alpha} \cup F\left(R^{\alpha}\right)$, and $R^{\lambda}:=\bigcup_{\alpha<\lambda} R^{\alpha}$ for limit ordinals $\lambda$. The deflationary fixed point of $F$ is constructed in the dual way starting with $A^{k}$ as the initial stage and taking intersections at successor and limit ordinals.

Definition 3.1. Inflationary fixed-point logic (IFP) is obtained from FO by allowing formulae of the form $[\mathbf{i f p} R \boldsymbol{x} \cdot \varphi(R, \boldsymbol{x})](\boldsymbol{x})$ and $[\mathbf{d f p} R \boldsymbol{x} \cdot \varphi(R, \boldsymbol{x})](\boldsymbol{x})$, for arbitrary $\varphi$, defining the inflationary and deflationary fixed point of the operator induced by $\varphi$.

Model checking games for LFP. Let us recall the definitions of model checking games for least fixed-point logic LFP (the games for the modal $\mu$-calculus are analogous). Consider a sentence $\psi \in \mathrm{LFP}$ which we assume is in negation normal form and well-named, i.e. every fixed-point variable is bound only once.

The game $\mathcal{G}(\mathfrak{A}, \psi)$ is a parity game whose positions are subformulae of $\psi$ instantiated by elements of $\mathfrak{A}$, i.e. expressions $\varphi(\boldsymbol{a})$ such that $\varphi(\boldsymbol{x})$ is a subformula of $\psi$, and $\boldsymbol{a}$ a tuple of elements of $\mathfrak{A}$. Player 0 (Verifier) moves at positions associated with disjunctions and formulae $\exists y \varphi(\boldsymbol{a}, y)$. From a position $(\varphi \vee \vartheta)(\boldsymbol{a})$ she moves to either $\varphi(\boldsymbol{a})$ or $\vartheta(\boldsymbol{a})$ and from a position $\exists y \varphi(\boldsymbol{a}, y)$ she can move to any position $\varphi(\boldsymbol{a}, b)$ such that $b \in \mathfrak{A}$. In addition, Verifier is supposed to move at atomic false positions, i.e., at positions $R \boldsymbol{a}$ where $\boldsymbol{a} \notin R^{\mathfrak{A}}$ and $\neg R \boldsymbol{a}$ where $\boldsymbol{a} \in R^{\mathfrak{A}}$. However, these positions do not have successors, so Verifier loses at atomic false positions. Dually, Player 1 (Falsifier) moves at conjunctions and formulae $\forall y \varphi(\boldsymbol{a}, y)$, and loses at atomic true positions. The rules described so far determine the model checking game for FO-formulae $\psi$ and it is easily seen that Verifier has a winning strategy in this game $\mathcal{G}(\mathfrak{A}, \psi)$ starting at a position $\varphi(\boldsymbol{a})$ if, and only if, $\mathfrak{A} \models \varphi(\boldsymbol{a})$.

For formulae in LFP, we also have positions $[\mathbf{f p} T \boldsymbol{x} . \varphi](\boldsymbol{a})$ (where fp stands for either lfp or $\mathbf{g f p}$ ) and $T \boldsymbol{a}$, for fixed-point variables $T$. At these positions 
there is a unique move (by Falsifier, say) to $\varphi(\boldsymbol{a})$, i.e. to the formula defining the fixed point. The priority labelling assigns even priorities to gfp-atoms Ta and odd priorities to lfp-atoms $T \boldsymbol{a}$. Further, if $T, T^{\prime}$ are fixed-point variables of different kind with $T^{\prime}$ depending on $T$ (which means that $T$ occurs free in the formula defining $T^{\prime}$ ), then $T$-positions get lower priority than $T^{\prime}$-positions. The remaining positions, not associated with fixed-point variables, do not have a priority (or have the maximal one). As a result, the number of priorities in the model checking game equals the alternation depth of the fixed-point formula plus one. For more details and explanations, and for the proof that the construction is correct, see e.g. $[3,8]$.

Theorem 3.2. $\mathfrak{A} \models \psi$ if, and only if, Verifier has a winning strategy for the parity game $\mathcal{G}(\mathfrak{A}, \psi)$ from position $\psi$.

Games for IFP. We restrict attention to finite structures. The model checking game for an IFP-formula $\psi$ on a finite structure $\mathfrak{A}$ is a backtracking game $\mathcal{G}(\mathfrak{A}, \psi)=\left(V, E, V_{0}, V_{1}, B, \Omega\right)$. As in the games for LFP, the positions are subformulae of $\psi$, instantiated by elements of $\mathfrak{A}$. We only describe the modifications.

We always assume that formulae are in negation normal form, and write $\bar{\vartheta}$ for the negation normal form of $\neg \vartheta$. Consider any ifp-formula $\varphi^{*}(\boldsymbol{x}):=$ $[\operatorname{ifp} T \boldsymbol{x} . \varphi(T, \boldsymbol{x})](\boldsymbol{x})$ in $\psi$. In general, $\varphi$ can have positive or negative occurrences of the fixed point variable $T$. We use the notation $\varphi(T, \bar{T})$ to separate positive and negative occurrences of $T$. To define the set of positions we include also all subformulae of $T \boldsymbol{x} \vee \varphi(T, \boldsymbol{x})$ and $\bar{T} \boldsymbol{x} \wedge \bar{\varphi}(T, \boldsymbol{x})$. From a position $\varphi^{*}(\boldsymbol{a})$ the play proceeds to $T \boldsymbol{a} \vee \varphi(T, \boldsymbol{a})$. When a play reaches a position $T \boldsymbol{c}$ or $\bar{T} \boldsymbol{c}$ the play proceeds back to the formula defining the fixed point by a regeneration move. More precisely, the regeneration of an ifp-atom $T \boldsymbol{c}$ is $T \boldsymbol{c} \vee \varphi(T, \boldsymbol{c})$, the regeneration of $\bar{T} \boldsymbol{c}$ is $\bar{T} \boldsymbol{c} \wedge \bar{\varphi}(T, \boldsymbol{c})$. Verifier can move from $T \boldsymbol{c}$ to its regeneration, Falsifier from $\bar{T} \boldsymbol{c}$. For dfp-subformulae $\vartheta^{*}(\boldsymbol{x}):=[\mathbf{d f p} R \boldsymbol{x} . \vartheta(R, \boldsymbol{x})](\boldsymbol{x})$, dual definitions apply. Verifier moves from $\bar{R} \boldsymbol{c}$ to its regeneration $\bar{R} \boldsymbol{c} \vee \bar{\vartheta}(R, c)$, and Falsifier can make regeneration moves from $R \boldsymbol{c}$ to $R \boldsymbol{c} \wedge \vartheta(R, \boldsymbol{c})$. The priority assignment associates with each ifp-variable $T$ an odd priority $\Omega(T)$ and with each dfp-variable $R$ an even priority $\Omega(R)$, such that for any two distinct fixed point variables $S, S^{\prime}$, we have $\Omega(S) \neq \Omega\left(S^{\prime}\right)$, and whenever $S^{\prime}$ depends on $S$, then $\Omega(S)<\Omega\left(S^{\prime}\right)$. Positions of the form $S \boldsymbol{c}$ and $\bar{S} \boldsymbol{c}$ are called $S$-positions. All $S$-positions get priority $\Omega(S)$, all other formulae get a higher priority. The set $B$ of backtrack positions is the set of $S$-positions, where $S$ is any fixed-point variable.

For simplicity we focus on IFP-formulae with a single fixed point, $\psi:=$ $[\operatorname{ifp} T \boldsymbol{x} \cdot \varphi](\boldsymbol{a})$ where $\varphi(T, \boldsymbol{x})$ is a first-order formula. When the play reaches a position $T \boldsymbol{c}$ Verifier can make a regeneration move to $T \boldsymbol{c} \vee \varphi(T, \boldsymbol{c})$ or backtrack. Dually, Falsifier can regenerate from positions $\bar{T} c$ or backtrack. However, since we have only one fixed point, all backtrack positions have the same priority and only one backtrack move can occur in a play.

In this simple case, the rules of the backtracking game ensure that infinite plays (which are plays without backtracking moves) are won by Falsifier, since 
ifp-atoms have odd priority. However, if one of the players backtracks after the play has gone through $\alpha T$-positions, then the play ends when $\alpha$ further $T$ positions have been visited. Falsifier has won, if the last of these is of form $T \boldsymbol{c}$, and Verifier has won if it is of form $\bar{T} \boldsymbol{c}$.

The differences between IFP model checking and LFP model checking are in fact best illustrated with this simple case. For this reason, and for lack of space, we prove the correctness of the model checking game only for this case, and defer the general case to the full version of this paper.

We claim that Verifier has a winning strategy for the game $\mathcal{G}(\mathfrak{A}, \psi)$ if $\mathfrak{A} \models \psi$ and Falsifier has a winning strategy if $\mathfrak{A} \not \neq \psi$.

To prove our claim, we look at the first-order formulae $\varphi^{\alpha}$ defining the stages of the induction. Let $\varphi^{0}(\boldsymbol{a})=$ false and $\varphi^{\alpha+1}(\boldsymbol{a})=\varphi^{\alpha}(\boldsymbol{a}) \vee \varphi\left[T / \varphi^{\alpha}, \bar{T} / \bar{\varphi}^{\alpha}\right](\boldsymbol{x})$. On finite structures $\psi(\boldsymbol{a}) \equiv \bigvee_{\alpha<\omega} \varphi^{\alpha}(\boldsymbol{a})$. Consider the situation after a backtracking move prior to which $\beta T$-positions have been visited and suppose that $\mathfrak{A} \models \varphi^{\beta}(\boldsymbol{a})$. A winning strategy for Verifier in the first-order game $\mathcal{G}\left(\mathfrak{A}, \varphi^{\beta}(\boldsymbol{a})\right)$ (from position $\varphi^{\beta}(\boldsymbol{a})$ ) translates in the obvious way into a (non-positional) strategy for the game $\mathcal{G}(\mathfrak{A}, \psi)$ from position $\psi(\boldsymbol{a})$ with the following properties: Any play that is consistent with this strategy will either be winning for Verifier before $\beta T$-positions have been seen, or the $\beta$-th $T$-position will be negative.

Similarly, if $\mathfrak{A} \not \models \varphi^{\beta}(\boldsymbol{a})$ then Falsifier has a winning strategy for $\mathcal{G}\left(\mathfrak{A}, \varphi^{\beta}(\boldsymbol{a})\right)$, and this strategy translates into a strategy for the game $\mathcal{G}(\mathfrak{A}, \psi)$ by which Falsifier forces the play (after backtracking) from position $\psi(\boldsymbol{a})$ to a positive $\beta$-th $T$-position, unless she wins before $\beta T$-positions have been seen.

Lemma 3.3. Suppose that a play on $\mathcal{G}(\mathfrak{A}, \psi)$ has been backtracked to the initial position $\psi(\boldsymbol{a})$ after $\beta$ T-positions have been visited. Verifier has a winning strategy for the remaining game if, and only if, $\mathfrak{A} \models \varphi^{\beta}(\boldsymbol{a})$.

From this we obtain the desired result.

Proposition 3.4. If $\mathfrak{A} \models \psi(\boldsymbol{a})$, then Verifier wins the game $\mathcal{G}(\mathfrak{A}, \psi(\boldsymbol{a}))$ from position $\psi(\boldsymbol{a})$. If $\mathfrak{A} \forall \forall(\boldsymbol{a})$, then Falsifier wins the game $\mathcal{G}(\mathfrak{A}, \psi(\boldsymbol{a}))$ from position $\psi(\boldsymbol{a})$.

Proof. Suppose first that $\mathfrak{A} \models \psi(\boldsymbol{a})$. Then there is some ordinal $\alpha<\omega$ such that $\mathfrak{A} \models \varphi^{\alpha}(\boldsymbol{a})$. We construct a winning strategy for Verifier in the game $\mathcal{G}(\mathfrak{A}, \psi(\boldsymbol{a}))$ starting at position $\psi(\boldsymbol{a})$.

From $\psi(\boldsymbol{a})$ the game proceeds to $(T \boldsymbol{a} \vee \varphi(\boldsymbol{a}))$. At this position, Verifier repeatedly chooses the node $T \boldsymbol{a}$ until this node has been visited $\alpha$-times. After that, she backtracks and moves to $\varphi(\boldsymbol{a})$. By Lemma 3.3 and since $\mathfrak{A} \models \varphi^{\alpha}(\boldsymbol{a})$, Verifier has a strategy to win the remaining play.

Now suppose that $\mathfrak{A} \not \forall \psi(\boldsymbol{a})$. If, after $\alpha T$-positions, one of the players backtracks, then Falsifier has a winning strategy for the remaining game, since $\mathfrak{A} \forall \forall \varphi^{\alpha}(\boldsymbol{a})$. Hence, the only possibility for Verifier to win the game in a finite number of moves is to avoid positions $\bar{T} \boldsymbol{b}$ where Falsifier can backtrack.

Consider the formulae $\varphi_{f}^{\alpha}$, with $\varphi_{f}^{0}=$ false and $\varphi_{f}^{\alpha+1}(\boldsymbol{x})=\varphi\left[\varphi_{f}^{\alpha}\right.$, false $](\boldsymbol{x})$. They define the stages of $[\mathbf{i f p} T \boldsymbol{x} \cdot \varphi[T$, false $](\boldsymbol{x})]$, obtained from $\psi$ by replacing negative occurrences of $T$ by false. If Verifier could force a finite winning play, 
with $\alpha-1$ positions of the form $T \boldsymbol{c}$ and without positions $\bar{T} \boldsymbol{c}$, then she would in fact have a winning strategy for the model checking game $\mathcal{G}\left(\mathfrak{A}, \varphi_{f}^{\alpha}(\boldsymbol{a})\right)$. Since $\psi_{f}^{\alpha}$ implies $\varphi^{\alpha}$, it would follow that $\mathfrak{A} \models \varphi^{\alpha}(\boldsymbol{a})$. But this is impossible.

\section{Definability of Backtracking Games}

In the previous section we demonstrated that backtracking games can be used as model-checking games for IFP. The aim of this section is to show that they are, in some sense, the "right" model-checking games for inflationary fixed-point logics. For this, we identify a natural sub-class of backtracking games, which we call simple, such that for every formula $\varphi \in \operatorname{IFP}$ and finite structure $\mathfrak{A}$, the game $\mathcal{G}(\mathfrak{A}, \varphi)$ can be trivially modified to fall within this class and, on the other hand, for every $k \in \mathbb{N}$ there is a formula $\varphi \in$ IFP defining the winning region for Player 0 in any simple game with at most $k$ priorities. In this sense, simple backtracking games precisely capture IFP model-checking.

Consider again the proof given in Section 3 for winning strategies in a game $\mathcal{G}(\mathfrak{A}, \varphi)$ and the way backtracking was used there: If Player 0 wanted to backtrack it was always after opening a fixed point, say [ifp $R \boldsymbol{x} . R \boldsymbol{x} \vee \varphi$ ]. She then looped $\alpha$ times through the $R \boldsymbol{x}$ sub-formula and backtracked. With choosing the $\alpha$ she essentially picked a stage of the fixed-point induction on $\varphi$ and claimed that $\boldsymbol{x} \in \varphi^{\alpha}$. From this observation we can derive two important consequences. As every inflationary fixed-point induction must close after polynomially many steps in the size of the structure $\mathfrak{A}$ and therefore in linearly many steps in terms of the game graph, there is no need for Player 0 to backtrack more than $n$ steps, where $n$ is the size of the game graph. Further, the game can easily be modified such that instead of having the nodes for the disjunction $R \boldsymbol{x} \vee \varphi$ and the sub-formula $R \boldsymbol{x}$, we simply have a node for $\varphi$ with a self-loop. In this modified game graph, not only is it sufficient for Player 0 to backtrack no more than $n$ steps, we can, in addition, require that whenever she backtracks from a node $v$, it must be to $v$ again, i.e. when she decides to backtrack from a node corresponding to the formula $\varphi$, she loops $\alpha$ times through $\varphi$ and then backtracks $\alpha$ steps to $\varphi$ again. The same is true for Player 1 and her backtracking.

Definition 4.1. A strategy in a backtracking game $\mathcal{G}$ is local if, for any backtracking node $v$, all backtracking moves from $v$ are to a previous occurrence of $v$. Given a function $f: \mathbb{N} \rightarrow \mathbb{N}$, we call a strategy $f$-backtracking if all backtracking moves made by the strategy have distance at most $f(|\mathcal{G}|)$. The strategy is called linear in case $f(n)=n$ and polynomial if $f$ is a polynomial in $n$.

As explained above, we can easily modify the construction of the game graph $\mathcal{G}(\mathfrak{A}, \varphi)$ for a formula $\varphi$ and structure $\mathfrak{A}$ such that every node in $B$ has a self loop. We call such game graphs inflationary.

Definition 4.2. A backtracking game $\mathcal{G}:=\left(V, E, V_{0}, V_{1}, B, \Omega\right)$ is inflationary, if every node in $B$ has a self-loop. An inflationary game $\mathcal{G}$ is called simple if both players have local linear winning strategies on their winning regions. 
Proposition 4.3. For any IFP-formula $\psi$ and every finite structure $\mathfrak{A}$, the model-checking game $\mathcal{G}(\mathfrak{A}, \varphi)$, as defined in Section 3, is simple.

We will construct IFP-formulae defining the winning regions of simple backtracking games. Since backtracking games are extensions of parity games we start with the formula defining winning regions in parity games (see [9]). Let $\mathcal{G}$ be a parity game with $k+1$ priorities and consider the formula $\varphi(x):=$ $\left[\operatorname{gfp} R_{0} x . \operatorname{lfp} R_{1} x \ldots \mathbf{f p} R_{k} x . \vartheta\left(x, R_{0}, \ldots, R_{k}\right)\right](x)$, where

$$
\begin{aligned}
\vartheta\left(x, R_{0}, \ldots, R_{k}\right):= & \bigwedge_{i=0}^{k}\left(V_{0} x \wedge \Omega(y)=i \rightarrow \exists y\left(E x y \wedge R_{i} y\right)\right) \wedge \\
& \bigwedge_{i=0}^{k}\left(V_{1} x \wedge \Omega(y)=i \rightarrow \forall y\left(E x y \rightarrow R_{i} y\right)\right) .
\end{aligned}
$$

For every node $v \in V$, we have that $\mathcal{G} \models \varphi(v)$ if, and only if, Player 0 has a winning strategy for the game $\mathcal{G}$ from $v$. A simple way to see this is to analyse the model checking game for $\varphi(v)$ on $\mathcal{G}$. If we remove the edges which would force a player to lose immediately, we obtain $\mathcal{G}$ itself (from position $v$ ).

We take this formula as a starting point for defining an IFP-formula deciding the winner of backtracking games. To define strategies involving backtracking, we first need some preparation. In particular, in order to measure distances we need an ordering on the arenas.

It is easily seen that backtracking games are invariant under bisimulation. Thus, it suffices to consider arenas where no two distinct nodes are bisimilar (we refer to such arenas as bisimulation minimal). The next step is to define an ordering on the nodes in an arena. This is done by ordering the bisimulation types realised in it.

Lemma 4.4. There is a formula $\varphi_{\text {ord }}(x, y) \in \mathrm{IFP}$ defining on every bisimulation minimal arena a linear order.

This is well-known in finite model theory. For an explicit construction, see e.g. [4]. As a result, we can assume that the backtracking games are ordered and that we are given an arithmetical predicate for addition with respect to the order defined above.

In Theorem 2.5 we exhibited a backtracking game that requires infinite memory strategies. All strategies in this game are necessarily local strategies. Thus Theorem 2.5 also applies to games with local strategies. The reason for the increased memory consumption is that when the decision to backtrack is made, it is necessary to know which nodes have been seen in the past, i.e. to which node a backtracking move is possible. However, since we here consider strategies with local backtracking only, it suffices to know the backtracking moves that are still active, i.e. have not yet been released. Thus we can capture all the relevant information about a partial play $\pi$ ending in position $v$ by the tuple $\left(v, d_{\pi}(0), \ldots, d_{\pi}(k)\right)$. This is formalised in the notion of a configuration.

Definition 4.5. Let $\mathcal{G}$ be a backtracking game with $k+1$ priorities. A configuration is a pair $(v, \boldsymbol{d})$ consisting of a node $v$ and a tuple $\boldsymbol{d} \in\{0, \ldots, k, \infty\}^{k+1}$. Let $\pi$ be a (partial) play ending in node $v$. The configuration of $\pi$ is the tuple $\left(v, d_{0}, \ldots, d_{k}\right)$ such that $d_{i}:=d_{\pi}(i)$ for all $i \leq k$. 
We are now ready to present a formula defining the winning region for Player 0 in a simple backtracking game with priorities $0, \ldots, k$. The structure of the formula is similar to the structure of $\varphi(x)$ for parity games, in the sense that for games with $k+1$ priorities we have $k+1$ nested fixed points of the form gfp $R_{0} x \boldsymbol{d}$. Ifp $R_{1} x \boldsymbol{d} \ldots$ fp $R_{k} x \boldsymbol{d}$ and a $\psi$ which is first-order, up to the IFPsubformula defining the order of the bisimulation types. In its various nested fixed points the formula builds up sets of configurations $\left(x, d_{0}, \ldots, d_{k}\right)$ such that if $\left(x, d_{0}, \ldots, d_{k}\right) \in R_{\Omega(x)}$, then Player 0 can extend any partial play $\pi$, ending in node $x$ with $d_{\pi}(j)=d_{j}$ for all $0 \leq j \leq k$, to a winning play. As the $d_{i}$ range over 0 to $n:=|\mathcal{G}|$ and also may take the value $\infty$, we would, strictly speaking, need to encode each $d_{i}$ by a pair of elements. However, to simplify notation, we only use one variable for each $d_{i}$ and allow it to take all possible values. We also use a constant $\infty$ and variables $i, j, \ldots$ for constants between 0 and $k$. Finally, in the case distinctions below we write $d_{i}=m$ for $\exists m \in\{0, \ldots, n\} \wedge d_{i}=m$.

The inner formula $\psi$ is split in two parts $\psi_{0} \vee \psi_{1}$ taking care of positions where Player 0 moves and positions where Player 1 moves. We first present the formula $\psi_{0}\left(x, R_{0}, \ldots, R_{k}\right)$ defining positions in $V_{0}$ from which Player 0 can win.

$$
\begin{aligned}
& \psi_{0}(x, \boldsymbol{d}):=V_{0} x \wedge \bigvee_{\mathrm{i}} \Omega(x)=\mathrm{i} \wedge \wedge_{l=i+1}^{k} d_{l}=\infty \wedge \\
& \exists y \exists \boldsymbol{d}^{\prime} E x y \wedge \bigvee_{j} \Omega(y)=\mathrm{j} \wedge R_{j} y \boldsymbol{d}^{\prime} \wedge \\
& d_{i}=\infty \wedge j \geq i \wedge \boldsymbol{d}=\boldsymbol{d}^{\prime} \vee \\
& \mathrm{j}<\mathrm{i} \wedge \boldsymbol{d}^{\prime}=\left(d_{0}, \ldots, d_{j}, \infty, \ldots, \infty\right) \vee \\
& B x \wedge \exists m \neq \infty R_{i}\left(x, d_{0}, \ldots, d_{i-1}, m, \infty, \ldots, \infty\right) \vee \\
& d_{i}=m \wedge \mathrm{j}>\mathrm{i} \wedge \boldsymbol{d}^{\prime}=\boldsymbol{d} \vee \\
& \mathrm{j}=\mathrm{i} \wedge \boldsymbol{d}^{\prime}=\left(d_{0}, \ldots, d_{i-1}, d_{i}-1, \infty, \ldots, \infty\right) \vee \\
& \mathrm{j}<\mathrm{i} \wedge \boldsymbol{d}^{\prime}=\left(d_{0}, \ldots, d_{j}, \infty, \ldots, \infty\right)
\end{aligned}
$$

The first line of the formula states that $x$ has to be in $V_{0}$, the priority of $x$ is $i$, for some $i$, and the tuple $\left(d_{0}, \ldots, d_{k}\right)$ has $\infty$ at all positions greater than $i$. This corresponds to the fact that a node of priority $i$ releases all backtracking moves on higher priorities. Now, Player 0 can win from configuration $(x, \boldsymbol{d})$ if she can move to a successor $y$ of $x$ from which she wins the play. That she can win from $y$ means that if $\left(y, \boldsymbol{d}^{\prime}\right)$ is the configuration reached when she moves from $(x, \boldsymbol{d})$ to $y$, then $\left(y, \boldsymbol{d}^{\prime}\right) \in R_{\Omega(y)}$. The second row of the formula states the existence of such a successor $y$ and the rest of the formula defines what it means for $\left(y, \boldsymbol{d}^{\prime}\right)$ to be the configuration reached from $x$ when moving to $y$.

The next formula $\psi_{1}$ takes care of nodes $x \in V_{1}$.

$$
\begin{aligned}
& \psi_{1}(x, \boldsymbol{d}):=V_{1} x \wedge \bigvee_{\mathrm{i}} \Omega(x)=\mathrm{i} \wedge \bigwedge_{l=i+1}^{k} d_{l}=\infty \wedge \\
& \left(B x \rightarrow \forall m<\infty R_{i}\left(x, d_{0}, \ldots, d_{i-1}, m, \infty, \ldots, \infty\right)\right) \wedge \\
& \forall y\left(E x y \rightarrow \bigvee_{j} \Omega(y)=\mathrm{j} \wedge \exists \boldsymbol{d}^{\prime} R_{j} y \boldsymbol{d}^{\prime} \wedge\right. \\
& d_{i}=\infty \wedge j \geq i \wedge \boldsymbol{d}^{\prime}=\left(d_{0}, \ldots, d_{i}, \infty, \ldots, \infty\right) \vee \\
& \mathrm{j}<\mathrm{i} \wedge \boldsymbol{d}^{\prime}=\left(d_{0}, \ldots, d_{j}, \infty, \ldots, \infty\right) \vee \\
& d_{i}=m \wedge j>i \wedge \boldsymbol{d}^{\prime}=\boldsymbol{d} \vee \\
& \mathrm{j}=\mathrm{i} \wedge \boldsymbol{d}^{\prime}=\left(d_{0}, \ldots, d_{i-1}, d_{i}-1, \infty, \ldots, \infty\right) \vee \\
& \mathrm{j}<\mathrm{i} \wedge \boldsymbol{d}^{\prime}=\left(d_{0}, \ldots, d_{j}, \infty, \ldots, \infty\right) \vee \\
& m=0 \wedge B x)
\end{aligned}
$$


A node $x \in V_{1}$ with configuration $(x, \boldsymbol{d})$ is good for Player 0 if Player 1 has no choice but to move to a node from which Player 0 wins. The formula is defined similarly to $\psi_{0}$ only that in the second line we ensure that if $x \in B$ then Player 0 must win the $m$-step game from $x$ for all $m$, as otherwise Player 1 could make a backtracking move and win, and further Player 0 now also wins the $m$-step game from $x$ where $m=0$.

With $\psi_{0}$ and $\psi_{1}$ defined we can present the formula $\varphi_{0}(x)$ true for a node $x$ in a simple backtracking game with $k+1$ priorities if, and only if, Player 0 has a linear winning strategy from $x$ with local backtracking.

$$
\varphi_{0}(x):=\left[\mathbf{g f p} R_{0} x \boldsymbol{d} . \mathbf{l f p} R_{2} x \boldsymbol{d} \ldots \text { fp } R_{k} x \boldsymbol{d} .\left(\psi_{0} \vee \psi_{1}\right)\right](x, \infty, \ldots, \infty)
$$

The next step is to show that the formula indeed defines the winning region for Player 0 . This is done by showing that whenever for a node $x$ the tuple $(x, \infty, \ldots, \infty)$ satisfies $\varphi_{0}$ then Player 0 has a winning strategy for the game starting at $x$.

It is a simple observation that the formula $\varphi_{1}$ defining the winning positions for Player 1 analogous to $\varphi_{0}$ is equivalent to the dual formula of $\varphi_{0}$. Thus, all nodes $x$ either satisfy $\varphi_{0}$ or $\varphi_{1}$ and therefore $\varphi_{0}$ defines the winning region for Player 0 and analogously $\varphi_{1}$ defines the winning region for Player 1 . This establishes the next theorem.

Theorem 4.6. Winning regions of simple backtracking games are definable in IFP.

Note that the definition of simple games involves semantic conditions, i.e. the players having linear strategies. It is open whether there is a purely syntactic criterion on game graphs allowing for the same kind of results.

\section{References}

1. A. Dawar, E. Grädel, and S. Kreutzer. Inflationary fixed points in modal logic. $A C M$ Transactions on Computational Logic (TOCL), 2003. Accepted for publication.

2. M. R. Garey and D. S. Johnson. Computers and Intractability. A Guide to the Theory of NP-Completeness. W. H. Freeman and company, New York, 1979. ISBN 0-7167-1044-7.

3. E. Grädel. Finite model theory and descriptive complexity. In Finite Model Theory and Its Applications. Springer-Verlag, 2003. To appear. See http://wwwmgi.informatik.rwth-aachen.de/Publications/pub/graedel/Gr-FMTbook.ps.

4. E. Grädel and S. Kreutzer. Will deflation lead to depletion? On non-monotone fixed-point inductions. In IEEE Symp. of Logic in Computer Science (LICS), 2003.

5. E. Grädel, W. Thomas, and T. Wilke (eds). Automata, Logics, and Infinite Games. A Guide to Current Research. Lecture Notes in Computer Science Nr. 2500, Springer, 2002.

6. S. Kreutzer. Expressive equivalence of least and inflationary fixed-point logic. In 17th Symp. on Logic in Computer Science (LICS), pages 403 - 413, 2002.

7. D. Martin. Borel determinacy, Annals of Mathematics 102 (1975), pp. 336-371.

8. C. Stirling. Bisimulation, model checking and other games. Notes for the Mathfit instructional meeting on games and computation. Edinburgh, 1997.

9. I. Walukiewicz. Monadic second order logic on tree-like structures. In STACS'96, volume 1046 of Lecture Notes in Computer Science (LNCS), pages 401 - 414. Springer Verlag, 1996. 